\title{
Dispositif pédagogique de formation à la lecture critique d'articles scientifiques utilisant une plate-forme numérique : impact sur les performances des étudiants à l'examen sommatif
}

\section{The use of an e-learning management system as an educational tool for scientific paper critical reading training: impact on students' performances at the final examination}

\section{Julien MANCINI, Roch GIORGI, Jean GAUDART, Jean-Charles Dufour} et Marius FIESCHI

Université d'Aix-Marseille, Faculté de médecine, Laboratoire d'Enseignement et de Recherche sur le Traitement de l'Information Médicale (LERTIM, EA 3283), 27 Bd Jean Moulin, 13385 Marseille, France

Assistance Publique - Hôpitaux de Marseille, Hôpital de la Timone, Service de Santé Publique et d'Information Médicale, 264 rue Saint Pierre, 13385 Marseille, France

Manuscrit reçu le 18 novembre 2008 ; commentaires éditoriaux formulés aux auteurs le 3 mars et le 28 avril 2009 ; accepté pour publication le 30 avril 2009

\author{
Mots clés : \\ Pédagogie médicale ; \\ lecture critique ; \\ plate-forme pédagogique \\ numérique ; \\ hybride
}

Résumé - Contexte : Depuis 2006/2007, l'enseignement de lecture critique d'articles (LCA) scientifiques en DCEM3 mis en place à la faculté de médecine de Marseille se déroule en mode hybride, avec l'aide, en amont et en aval du cours en présentiel, de la plate-forme numérique eCUME (e-Cursus à l'Université de la MEditerranée). Buts : Décrire l'utilisation par les étudiants de cette nouvelle plate-forme pédagogique et rechercher une association entre son utilisation et les scores obtenus à l'examen de LCA. Méthodes : Cette étude a inclus 275 étudiants inscrits pour la première fois en DCEM3 en 2007/2008 et présents à l'examen de LCA. Les traces de connexion concernant 91 ressources didactiques réparties en huit types informationnels ont été analysées. Résultats : Parmi les 275 étudiants, 195 ont accédé à au moins une des ressources étudiées (70,9\%). L'ensemble des étudiants ont consulté une médiane de 15 ressources (entre 0 et 150). Les quartiles d'utilisation de la plateforme pédagogique étaient significativement liés au score final sur $20(10,6 ; 10,7 ; 11,8 ; 11,6$ pour respectivement $0 ; 1$ à $15 ; 16$ à 39 et 40 à 150 consultations de ressources didactiques ; $p<0,001)$. Après ajustement sur le niveau général (scores hors LCA en DCEM3) et spécifique (score de LCA en DCEM2) de l'étudiant, ceux ayant accédé à plus de 15 ressources conservaient un meilleur score final $(p=0,001)$. Conclusion : L'utilisation, même débutante, par les étudiants d'une nouvelle plate-forme numérique pour la pédagogie et l'investissement pédagogique réalisé peuvent avoir un impact positif sur l'apprentissage de la lecture critique d'articles scientifiques. 


Keywords:
Medical education;
critical reading;
e-learning management
system;
hybrid

Abstract - Context: Since 2006/2007, teaching of scientific paper critical reading (SPCR) to 5th year medical students was introduced at the University of Marseille using a hybrid teaching approach combined with an e-learning management system method, the eCUME ("e-Cursus à l'université de la MEditerranée"). Objectives: To describe the use of this new e-learning platform by students, and to seek for an association between its use and students' scores at the scientific paper critical reading examination. Methods: This study comprised 275 students subscribed for the first time in the 5th year of medicine during 2007/2008 and attending at the SPCR examination. Archives of access related to 91 educational resources separated in eight informational types, were analyzed. Results: Among the 275 students, 195 accessed to at least one of the proposed resources (70.9\%). Majority of students has consulted a median of 15 resources (between 0 and 150). Quartiles of e-learning platform use were significantly associated to final score out of $20(10.6 ; 10.7 ; 11.8$; and 11.6 for respectively 0 ; 1 to $15 ; 16$ to 39 ; and 40 to 150 consulted educational resources: $p<0.001)$. After adjustment of student' general level (5th year student scores without SPCR) and specific level (4th year student' SPCR scores), those who accessed to more then 15 resources had a better final score ( $p=0.001$ ). Conclusion: The use (even naïve) of the new e-learning platform by students and the educational investment achieved could have a positive impact on scientific paper critical reading learning.

\section{Introduction}

La généralisation progressive de la mise à disposition des enseignants de plates-formes numériques pour la pédagogie amène à se poser la question de leur pertinence. L'intérêt de telles plates formes est au moins double : d'un côté, elles sont en mesure de faciliter l'accès des étudiants à de nombreuses ressources didactiques spécifiques ou sélectionnées sur Internet ; de l'autre, elles permettent d'envisager autrement les interactions entre les étudiants et les enseignants et sont susceptibles de les favoriser, en permettant notamment qu'elles se déroulent de manière asynchrone, ce qui permet théoriquement de s'affranchir de certaines contraintes. Pour autant, les conditions d'une authentique valeur ajoutée concernant l'impact de ces dispositifs sur les apprentissages des étudiants reste encore à documenter ${ }^{[1]}$. Il est acquis que l'utilisation de services numériques pour les activités d'enseignement et d'apprentissage est plus efficace que l'absence d'intervention pédagogique. Par contre, envisagée d'une manière globale, l'efficacité des dispositifs de formation médiés par les nouvelles technologies semble équivalente à celle des dispositifs d'enseignement traditionnel ${ }^{[1]}$. Il est désormais nécessaire que de futurs travaux clarifient à quelles conditions (quand et comment) l'emploi de ces nouvelles technologies serait susceptible de s'avérer efficient ${ }^{[2]}$. Il serait notamment utile de mieux connaître à quelles conditions le recours aux nouvelles technologies pour proposer un accès à diverses ressources didactiques en complément d'un enseignement traditionnel pourrait être bénéfique, par exemple en stimulant le travail personnel des apprenants.

La présente contribution présente un dispositif de formation à la lecture critique d'articles (LCA) de type hybride ${ }^{[3]}$, c'est-à-dire articulant des séances de formation en présentiel et des phases de formation à distance, soutenues par un environnement médiatisé par des technologies éducatives, développé à la faculté de médecine de l'université de la Méditerranée à Marseille (France). L'enseignement de la LCA s'inscrit dans la perspective actuelle de la médecine fondée sur les données probantes et doit permettre aux étudiants d'acquérir les compétences 
nécessaires pour comprendre et interpréter les résultats de la recherche médicale afin de pouvoir utiliser les meilleures preuves scientifiques du moment, pour une prise en charge personnalisée de chaque patient ${ }^{[4]}$. Dans cette perspective, qui implique la poursuite de l'apprentissage de la médecine tout au long d'une carrière, il est crucial d'acquérir un esprit critique vis-à-vis des publications ${ }^{[5,6]}$, mais il est tout aussi fondamental d'acquérir une autonomie de formation. Il s'agit également d'un enjeu pédagogique important pour les facultés de médecine françaises dans la mesure où l'épreuve de LCA sera introduite dans le cadre des épreuves classantes nationales (ECN) à partir de 2009.

Après l'acquisition des bases théoriques permettant d'appréhender les principes de la construction et de diffusion des connaissances scientifiques, les étudiants doivent apprendre à critiquer des articles et à répondre à des questions selon les objectifs publiés par le Centre national des concours d'internat $(\mathrm{CNCI})^{[7]}$. C'est dans le cadre de cette deuxième phase que le dispositif de formation rapporté a été développé. Il permet la mise à disposition des étudiants de multiples ressources et activités complémentaires accessibles à distance. Ce dispositif vise à stimuler le travail personnel des étudiants avant et après les séances en amphithéâtre et à focaliser ces séances sur la critique des articles et la compréhension des réponses aux questions plutôt que sur leur rédaction. Toutefois l'utilisation de la plateforme numérique et notamment la conception de documents didactiques ad hoc, voire la simple conception du cours en ligne avec la proposition de liens vers des ressources pertinentes, demande aux enseignants un investissement conséquent et implique la participation active des étudiants. Il est donc primordial de vérifier l'appropriation par les étudiants de ces nouveaux outils et d'évaluer leur impact sur les apprentissages.

L'objectif de cette étude était de décrire, à partir de l'exemple d'un module d'enseignement consacré à la LCA en cinquième année des études médicales (DCEM3), l'utilisation par les étudiants d'une nouvelle plate-forme pédagogique et de rechercher une association entre indicateurs d'utilisation et scores obtenus par les étudiants à l'examen sommatif correspondant.

\section{Matériel et méthodes}

L'environnement institutionnel en matière de technologies éducatives

Depuis l'année universitaire 2006/2007, l'université de la Méditerranée (Marseille, France) a mis en place un environnement numérique de travail (ENT) pour les étudiants, les enseignants et les personnels administratifs de l'université. Au sein de cet ENT, la plate-forme Moodle, baptisée eCUME dans le système d'information de l'université (e-Cursus à l'Université de la MEditerranée), offre aux étudiants et aux enseignants des services numériques pour la pédagogie. Cette plate-forme contient des cours, correspondant aux modules d'enseignement, mis en ligne et pourvus en ressources didactiques par les enseignants eux-mêmes. Un cours se présente sous la forme d'une page Internet divisée verticalement en trois parties : deux bandeaux latéraux présentent différentes fonctionnalités de base (étudiants inscrits au cours, dernières modifications des supports de cours, prochains évènements, liste des podcasts disponibles pour le cours, etc.) et une zone centrale (figure 1). Dans cette zone centrale les enseignants du module mettent à disposition des étudiants diverses ressources et activités didactiques de différentes natures (documents écrits, multimédias, forum, quiz, etc.) ainsi que la programmation des devoirs à rendre. Ces ressources peuvent être créées spécifiquement pour l'enseignement concerné mais peuvent aussi être des liens vers des documents didactiques pertinents disponibles sur Internet. Après connexion à l'ENT, à l'aide d'un login et mot de passe personnels, tous les étudiants inscrits administrativement à un (ou plusieurs) module(s) d'enseignement(s) peuvent accéder au(x) cours numérique(s) correspondant(s) sur eCUME. Ils disposent de plus de l'accès à la bibliothèque numérique de l'université et aux journaux scientifiques en ligne. 
THHK- UUNIVERSITÉ

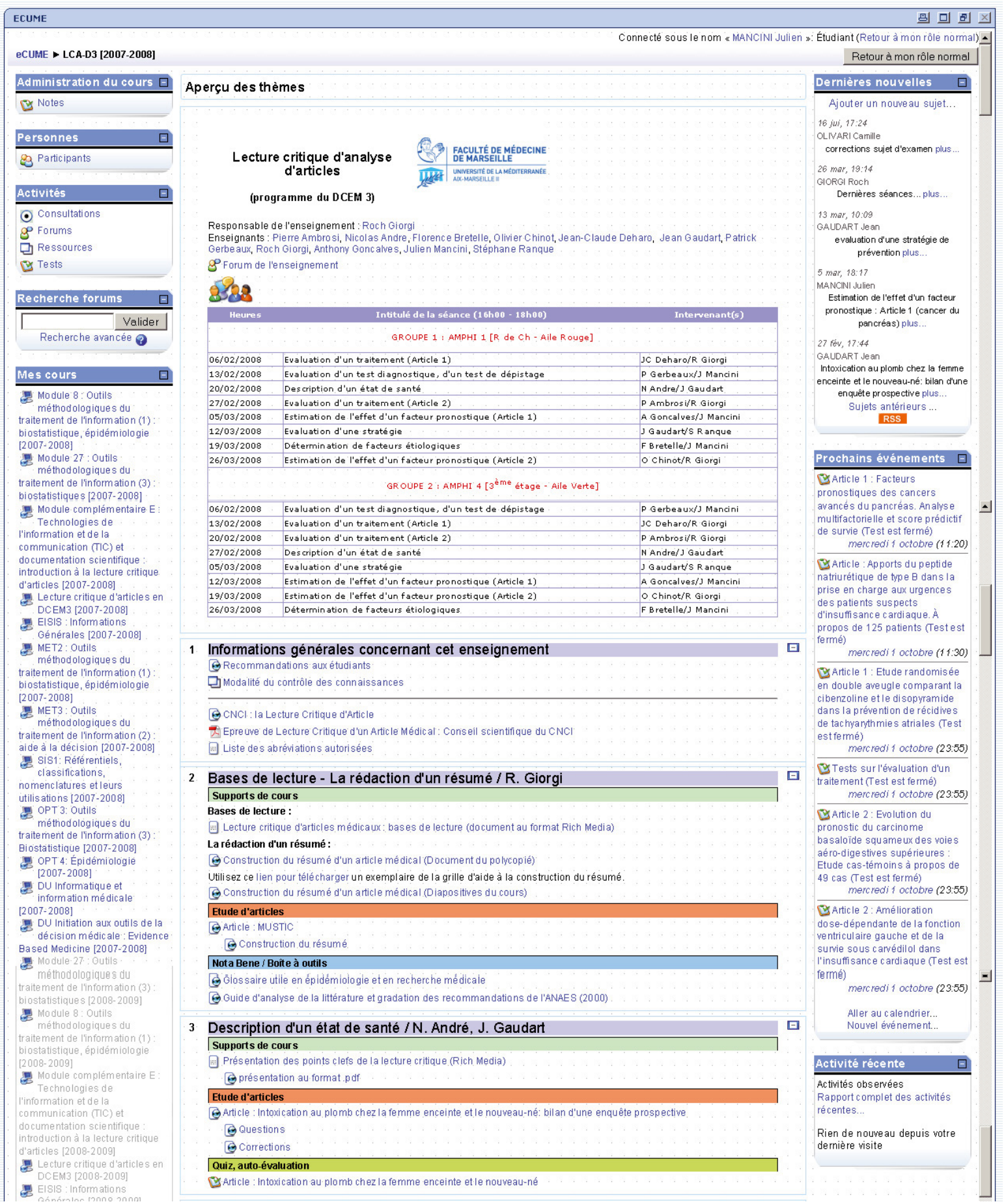

Fig. 1. Aperçu (affichage étudiant) du cours de lecture critique d'article sur la plate-forme eCUME. 
Le dispositif de formation à la lecture critique d'article mis en place à la faculté de médecine de Marseille

La faculté de médecine de Marseille a décidé la mise en place d'une formation à la LCA pluriannuelle au cours des quatre années du deuxième cycle des études médicales (DCEM) avec une différenciation des modalités d'enseignement (cours magistraux ou enseignement dirigés, en amphithéâtre ou en petits groupes) selon les années d'études.

Pour les étudiants en deuxième (DCEM2) et troisième (DCEM3) années du DCEM, l'enseignement de LCA a débuté en 2006/2007. En DCEM2, l'enseignement consiste en des enseignements dirigés, auprès de groupes d'étudiants en nombre restreint, fournissant aux étudiants les bases théoriques de la LCA selon les types d'études épidémiologiques (étude de cohorte comparative type essai clinique contrôlé, étude de cohorte exposés-non exposés, étude cas-témoins, etc.). En DCEM3, l'enseignement est davantage centré sur l'objectif principal de l'article et donc sur la question médicale concernée (évaluation de l'efficacité d'un traitement ou des capacités diagnostiques d'un test, recherche de facteurs étiologiques ou pronostiques, etc.). À partir d'un article de leur choix écrit en français ou dont la traduction française est disponible, des binômes associant un clinicien et un méthodologiste préparent une liste de questions explorant les objectifs publiés par le $\mathrm{CNCI}^{[7]}$ et proposent les réponses types attendues des étudiants (corrigés); ces binômes assurent les cours (huit séances de deux heures) en amphithêâtre par demi-promotion (d'environ 140 étudiants chacune). Au total, huit articles sont ainsi préparés.

En DCEM3, le dispositif d'enseignement de LCA mis en place peut-être qualifié d' "hybride", c'est-à-dire articulant des séances de formation en présentiel et des phases de formation à distance, soutenues par un environnement médiatisé par des technologies éducatives, de multiples ressources et activités étant mises à disposition des étudiants par l'intermédiaire de l'ENT et de la plate-forme eCUME (tableau I). En introduction de la première séance, le dispositif pédagogique mis en place est présenté aux étudiants. En fin de chaque séance, la mise à disposition de documents complémentaires correspondant à la séance est rappelée aux étudiants.

Préalablement aux cours en amphithéâtre, un polycopié (contenant les huit articles et les questions à traiter) est distribué aux étudiants. Les huit articles et les questions sont aussi disponibles via eCUME, ainsi qu'un quiz par article, constitué à partir des grilles de lecture d'articles médicaux proposées en 2000 par l'ANAES (agence nationale d'accréditation et d'évaluation en santé remplacée en 2005 par la haute autorité de santé, HAS) ${ }^{[8]}$. Ce quiz permet à l'étudiant de s'auto-évaluer en vérifiant sa capacité à identifier les principaux éléments méthodologiques pertinents concernant un article. Sont également accessibles des informations générales sur l'enseignement ainsi que des ressources didactiques complémentaires ${ }^{[9-11]}$. Ces dernières, produites par les enseignants de la faculté ou vérifiées par leurs soins, ont principalement pour objet des synthèses méthodologiques sur la LCA (construction d'un résumé d'article, guide d'analyse de la littérature, analyse d'un essai thérapeutique,...). Elles sont en nombre initialement limité afin de ne pas aboutir à une surcharge cognitive des étudiants avec des informations d'importance variable. Ces ressources sont évolutives et les questions des étudiants peuvent amener à ajouter de nouvelles ressources au cours de l'année.

En aval, après les séances correspondantes en amphithéâtre, diverses ressources didactiques numériques sont rendues accessibles aux étudiants via eCUME : les diapositives utilisées en amphithéâtre, une proposition de corrigé écrit aux questions posées et un diaporama sonorisé d'environ une demiheure reprenant les points clefs de la lecture critique. La mise en ligne de ces ressources répond à plusieurs objectifs différents. Les diapositives, supports de l'enseignement en amphithéâtre, permettent aux étudiants présents au cours de compléter leurs notes personnelles. Les corrigés écrits ont l'avantage par rapport à un polycopié de pouvoir être rendues accessibles aux étudiants dès la fin des séances en amphithéâtre. Enfin, les diaporamas sonorisés 


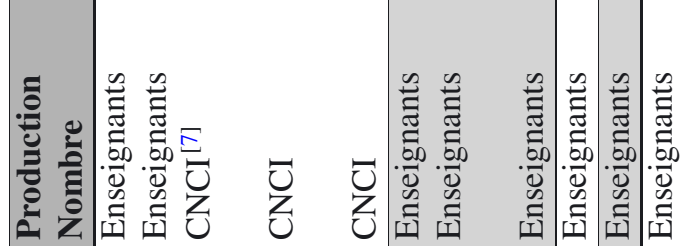

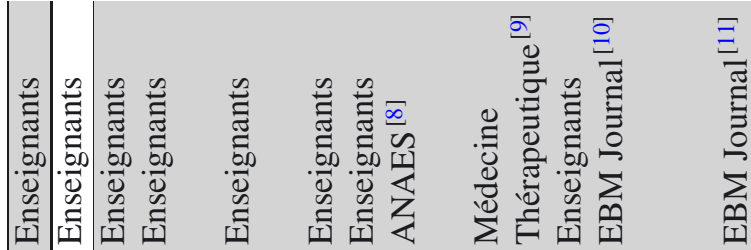

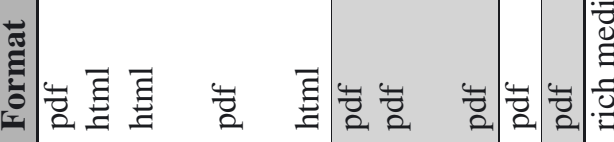

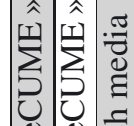

๕్

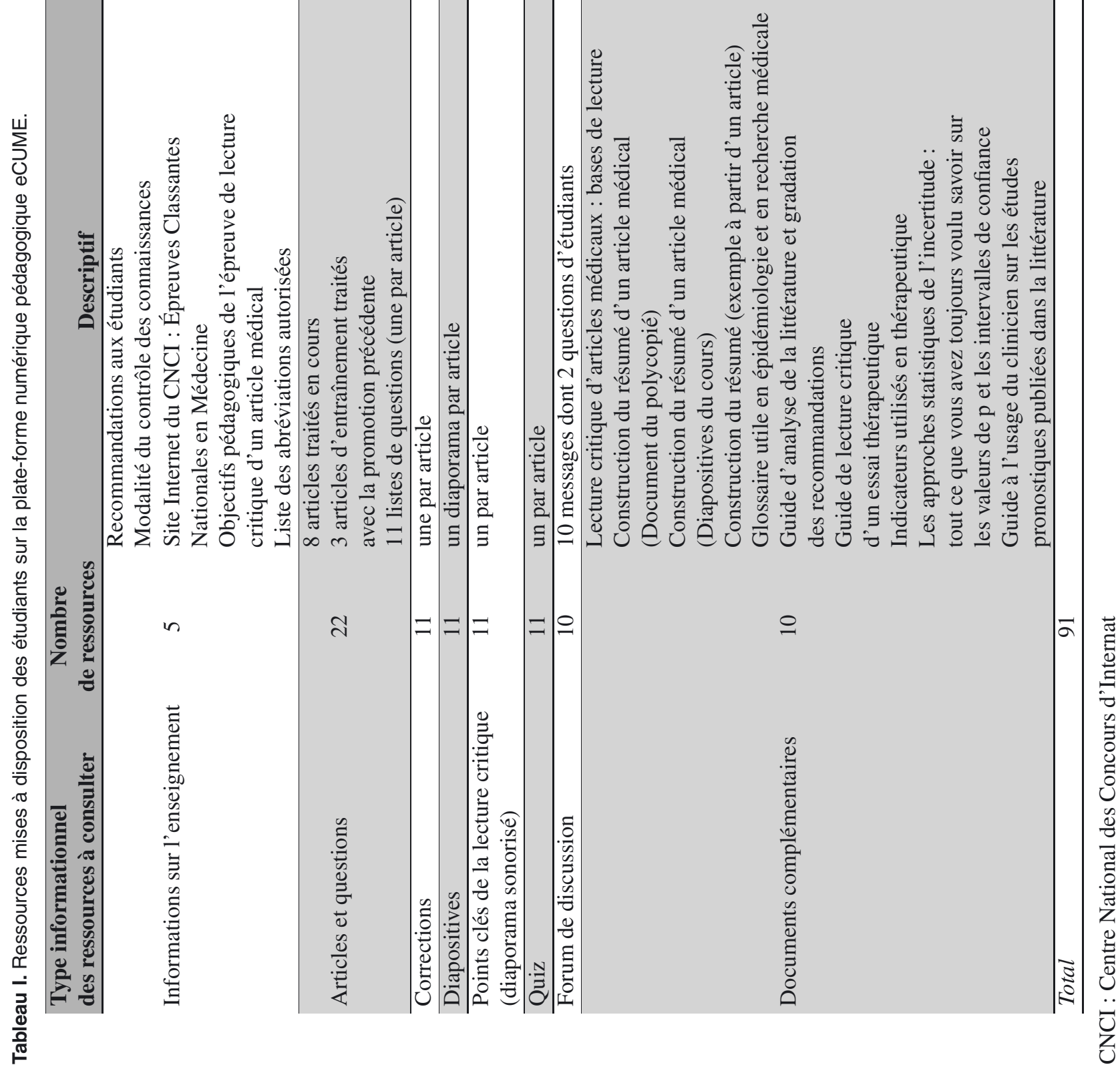


reproduisent les éléments essentiels du cours, qu'ils enrichissent de commentaires et explications de la part de l'enseignant, et peuvent être utilisés profitablement à distance de celui-ci, par exemple lors des révisions.

En plus de ces ressources, l'activation d'un forum de discussion pendant l'année universitaire de l'enseignement permet aux étudiants d'interagir entre eux et avec les enseignants à distance des cours.

Finalement, les différentes ressources utilisées peuvent être réparties en huit types informationnels (cf. tableau I, première colonne). Elles visent à accompagner et à stimuler le travail personnel asynchrone de l'étudiant avant et après la séance en amphithéâtre. Un autre objectif, à plus long-terme, est la constitution d'une banque d'articles pour les étudiants. En effet, chaque année universitaire, trois des huit articles sont renouvelés. Toutes les ressources concernant les articles traités antérieurement (articles, questions, corrections, diapositives, diaporama sonorisé, quiz) sont ainsi mises à disposition des étudiants qui souhaitent s'entraîner.

\section{Étudiants participants}

Les étudiants participants étaient les 287 étudiants inscrits en DCEM3 à la faculté de médecine de Marseille (université de la Méditerranée) lors de l'année universitaire 2007/2008. Afin d'étudier les liens entre l'utilisation de la plate-forme pédagogique numérique et les scores aux examens, n'ont été inclus que les étudiants inscrits pour la première fois en DCEM3 et s'étant présentés à la première session de l'examen de LCA en mai 2008.

\section{Traces de connexion}

Les données utilisées pour cette étude étaient les traces de connexion, c'est-à-dire les différents types d'actions effectuées par les utilisateurs d'un serveur, par exemple : connexion, déconnexion, mais aussi clic sur un lien hypertexte identifié, temps de consultation d'une ressource, etc. L'ensemble des traces de connexion des étudiants au cours de LCA de DCEM3 au sein d'eCUME ont été recueillies du début des cours (second quadrimestre) jusqu'à la veille de l'examen de la première session. La connexion au cours de LCA de DCEM3 était réservée aux seuls étudiants inscrits administrativement et pédagogiquement et nécessitait une connexion à l'ENT avec identification de l'étudiant et saisie de son mot de passe personnel.

Les actions suivantes, enregistrées automatiquement dans les traces de connexion, ont été analysées :

- la consultation du « cours », c.-à-d. la connexion individuelle au cours de LCA mis en ligne sur la plate-forme,

- la consultation d'une ressource, c.-à-d. chaque clic permettant d'accéder à une des ressources didactiques mise à disposition sur la plate-forme.

Les 91 ressources auxquelles les étudiants pouvaient accéder en cliquant sur un des liens du cours étaient réparties en huit types informationnels (tableau I).

Afin de quantifier l'utilisation du cours sur la plate-forme numérique, deux types d'indicateurs ont été utilisés :

- la variété de l'utilisation : accès aux différents types informationnels de ressources,

- la répétition de l'utilisation : nombre d'accès (clics) aux ressources.

Scores aux examens

Les scores obtenus par les étudiants lors de la première session des examens de DCEM3 ont été recueillis (scores au module de LCA et total des scores à l'ensemble des modules). De plus, nous avons recueilli les scores obtenus par ces étudiants l'année précédente, en DCEM2, au module de LCA. L'examen de LCA en DCEM3 (épreuve rédactionnelle de trois heures) étant noté sur 40, les scores ont été ramenés sur 20 points afin de faciliter l'interprétation et les comparaisons avec les scores de LCA obtenus en DCEM2 (examen noté sur 20 points). Ainsi, un score supérieur ou égal à 10 permettait de valider le 
module. Un score inférieur à huit était éliminatoire et nécessitait de se présenter à la session de rattrapage quels que soient les scores obtenus aux autres modules. Afin d'avoir un indicateur du niveau général des étudiants dans les autres matières que la LCA, le score de LCA a été soustrait du total des scores à l'ensemble des modules en DCEM3. Le total hors LCA obtenu a ensuite été ramené sur 20 points.

\section{Analyses statistiques}

Les liens entre variables quantitatives étaient mesurés à l'aide du coefficient de corrélation non paramétrique de Spearman (rho). Afin de favoriser l'interprétation des résultats, en exprimant des scores moyens plutôt que des coefficients de corrélations, les indicateurs d'utilisation de la plate-forme ont été découpés en quartiles. Ce découpage empirique permettait d'identifier approximativement quatre profils d'utilisation : nulle, anecdotique, régulière et intensive. Les différences de scores en fonction des indicateurs de connexion, découpés en quartiles, ont été testées à l'aide d'analyses de variance univariées. Les scores obtenus à l'épreuve de LCA en DCEM3 ne variant pas de manière linéaire selon les indicateurs d'utilisation, nous n'avons conservé ensuite que deux groupes d'utilisateurs définis par rapport aux médianes des indicateurs. Des ajustements sur le niveau général (scores hors LCA en DCEM3) et spécifique (score de LCA en DCEM2) de l'étudiant ont alors été réalisés à l'aide d'analyses de variance multivariées.

Toutes les données ont été analysées sous SPSS 15.0. Les figures 2 et 3 (bubble charts) ont été réalisées à l'aide de R 2.3.1. Pour tous les tests bilatéraux utilisés, le seuil de significativité a été fixé à $5 \%$.

\section{Résultats}

Sur 287 étudiants inscrits en DCEM3 lors de l'année universitaire 2007/2008, neuf redoublants $(3,1 \%)$ ont été exclus de l'analyse ainsi que trois étudiants
$(1,0 \%)$ qui n'ont pas passé l'examen de LCA lors de la première session (deux absents à tous les examens, un absent à l'épreuve de LCA). Les analyses suivantes ont donc été réalisées sur 275 étudiants.

Les scores médians obtenus [score minimalscore maximal] aux premières sessions étaient : 12,0 [6-17] et 11,0 [5-16,5] pour les examens de LCA en DCEM2 et DCEM3 respectivement. La médiane pour l'ensemble des scores de DCEM3, à l'exception de celle de LCA, était 12,6 [4,5-16,7]. Les corrélations entre le score de LCA de DCEM3 et les autres scores recueillis étaient positives : 0,32 avec le score de LCA de DCEM2 ( $p<0,001) ; 0,39$ avec les autres scores de DCEM3 $(p<0,001)$.

Parmi les 275 étudiants inclus, 201 (73,1\%) se sont connectés au moins une fois individuellement au cours de LCA mis en ligne sur la plate-forme et 195 ont accédé à au moins une des ressources étudiées $(70,9 \%)$. L'ensemble des étudiants a consulté une médiane de 4 [0-8] types informationnels différents de ressources et de 15 [0-150] ressources. Sur les 195 étudiants ayant consulté au moins une ressource, ces indicateurs valaient respectivement 5 [1-8] et 26 [1-150].

Les huit types informationnels contenant un nombre différent de ressources disponibles, un ratio nombre d'accès moyen sur nombre de ressources a été calculé afin de pouvoir comparer leur utilisation (tableau II). Les types informationnels les plus consultés étaient alors les corrections écrites, suivis des documents complémentaires et des informations générales concernant l'enseignement.

Le nombre de types informationnels consultés et le nombre d'accès aux différentes ressources étaient positivement corrélés entre eux (rho $=0,92 ; p<$ $0,001)$. Après découpage de ces indicateurs en quartiles, les scores moyens obtenus étaient significativement différents en fonction du nombre de types informationnels consultés ( $p=0,001$; figure 2 ) et des accès aux différentes ressources $(p<0,001$; figure 3). Toutefois, l'effet de l'utilisation de la plateforme n'était pas linéaire.

Après ajustement sur le niveau général et spécifique de l'étudiant (évalués respectivement par les 
Tableau II. Accès aux ressources mises à disposition des étudiants ( $n=275)$ sur la plate-forme numérique pédagogique eCUME.

\begin{tabular}{lcccc}
\hline Type informationnel & $\begin{array}{l}\text { Nombre d'accès médian } \\
\text { [intervalle interquartile] }\end{array}$ & $\begin{array}{c}\text { Valeurs } \\
\text { extrêmes }\end{array}$ & $\begin{array}{c}\text { Nombre ressources } \\
\text { nombre de ressources }\end{array}$ & $\begin{array}{c}\text { Nombre d'accès } \\
\text { moyen / }\end{array}$ \\
\hline $\begin{array}{l}\text { Informations sur } \\
\text { l'enseignement }\end{array}$ & $0[0-3]$ & {$[0-17]$} & 5 & 0,37 \\
\hline Articles et questions & $1[0-4]$ & {$[0-37]$} & 22 & 0,20 \\
\hline Corrections & $3[0-11]$ & {$[0-57]$} & 11 & 0,57 \\
\hline Diapositives & $0[0-3]$ & {$[0-34]$} & 11 & 0,26 \\
\hline Points clés de la lecture critique & $1[0-3]$ & {$[0-31]$} & 11 & 0,23 \\
(diaporama sonorisé) & $0[0-1]$ & {$[0-7]$} & 11 & 0,06 \\
\hline Quiz & $0[0-0]$ & {$[0-11]$} & 10 & 0,07 \\
\hline Forum de discussion & $2[0-7]$ & {$[0-39]$} & 10 & 0,49 \\
\hline Documents complémentaires & $15[0-39]$ & {$[0-150]$} & 91 & 0,27 \\
\hline Total / 8 types informationnels & & & & \\
\hline
\end{tabular}

scores obtenus dans les autres matières que la LCA en DCEM3 et par le score de LCA en DCEM2), l'utilisation variée de la plate-forme pédagogique restait significativement liée au score de LCA en DCEM3 (moyennes marginales estimées : 11,0; 11,4 ; pour respectivement 0 à 4 et 5 à 8 types informationnels consultés ; $p=0,019$ ). De même, l'utilisation répétée de la plate-forme était toujours associée après ajustement à un meilleur score (moyennes marginales estimées : 10,$8 ; 11,5$ pour respectivement 0 à 15 et 16 à 150 documents didactiques consultés ; $p=0,001)$.

\section{Discussion}

Cette étude décrivant un dispositif pédagogique utilisant une plate-forme numérique dans le cadre d'un module d'enseignement à la faculté de médecine de Marseille, université de la Méditerranée, a montré une utilisation débutante de cet outil pour l'enseignement de la lecture critique d'articles scientifiques. La stimulation du travail personnel des étudiants dans le cadre d'un enseignement structuré selon un dispositif hybride, semble pourtant avoir un impact positif sur l'apprentissage.

Presque un tiers $(29,1 \%)$ des étudiants n'ont pas accédé aux ressources didactiques proposées. Par contre, parmi la large majorité des étudiants qui s'est connectée au moins une fois au cours après identification personnelle, la quasi-totalité a consulté au moins une des ressources. Les utilisateurs ont eu un nombre d'accès très variable aux 91 ressources (jusqu'à 150 accès), mais le nombre médian d'accès aux ressources (15) était largement inférieur au nombre de ressources proposées.

Nos résultats sont à rapprocher de ceux d'une étude conduite en 2004 chez 50 étudiants de DCEM3 et DCEM4 de la faculté de médecine Xavier Bichat dans laquelle il avait été mis en évidence que $26 \%$ d'entre eux n'avaient pas d'accès personnel à Internet et que plus de $45 \%$ l'utilisaient rarement pour leurs études du fait d'un manque d'information sur les sites à consulter ${ }^{[12]}$.

La situation concernant l'accès à Internet a certainement beaucoup évolué en quatre ans mais le déploiement, au sein d'un environnement numérique de travail, d'une plate-forme numérique 


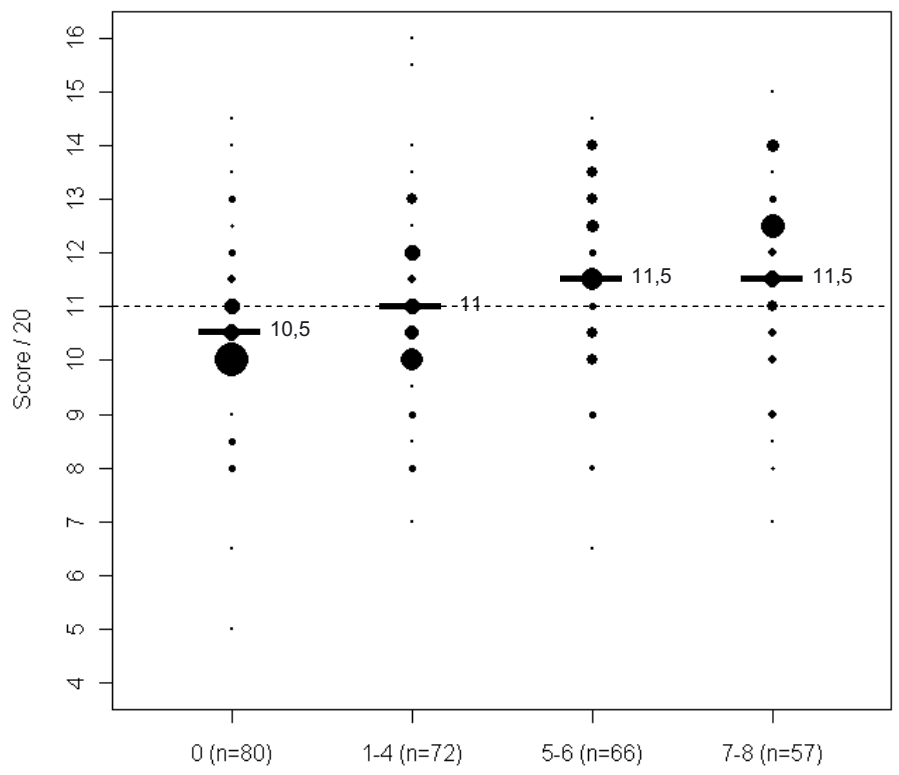

Type de ressources différentes consultées

Fig. 2. Scores des étudiants à la première session de LCA en DCEM3 en fonction des quartiles d'utilisation variée de la plate-forme pédagogique ( $p=0,001)$. Les cercles ont une surface proportionnelle au nombre d'étudiants ayant eu le score; les traits horizontaux gras indiquent la médiane des scores dans les différents groupes; le trait horizontal en pointillé indique la médiane générale $(11 ; N=275)$.

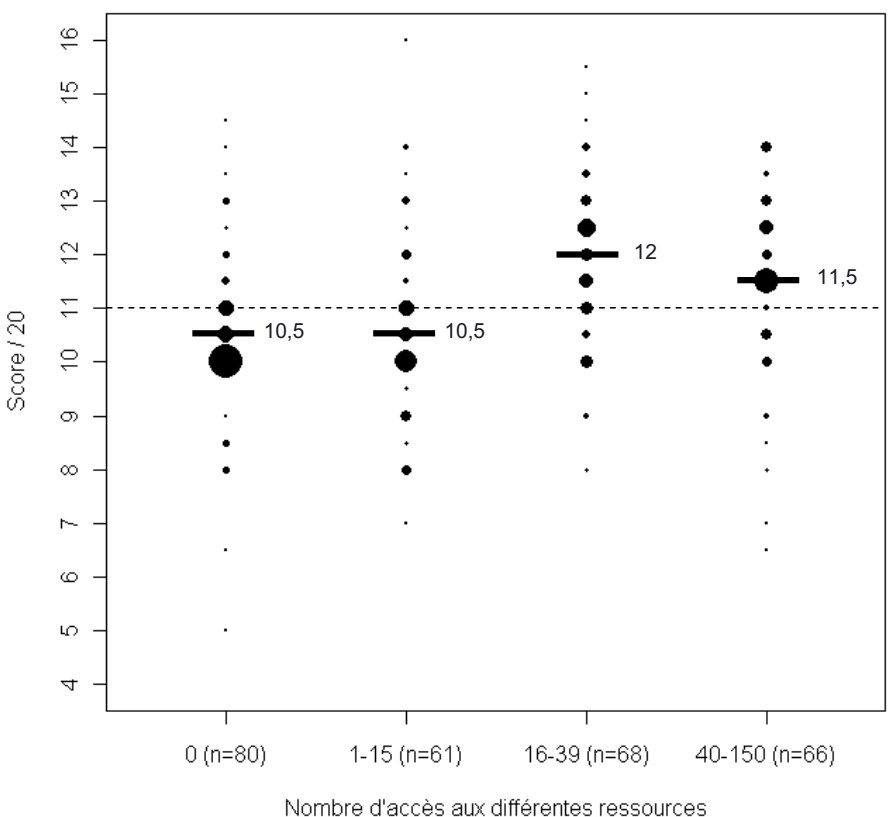

Fig. 3. Scores des étudiants à la première session de LCA en DCEM3 en fonction des quartiles d'utilisation répétée de la plate-forme pédagogique $(p<0,001)$. Les cercles ont une surface proportionnelle au nombre d'étudiants ayant eu le score; les traits horizontaux gras indiquent la médiane des scores dans les différents groupes; le trait horizontal en pointillé indique la médiane générale $(11 ; N=275)$.

LCA = lecture critique d'article

DCEM3 = année du deuxième cycle des études médicales 
pédagogique ne peut pas corriger les défauts d'accès à Internet. Il est probable que parmi les 29,1\% d'étudiants n'ayant jamais consulté nominativement une seule ressource, certains n'ont pas un accès pratique pour une utilisation pédagogique d'Internet. Bien que les mots de passe des étudiants soient personnels et donnent accès à l'ensemble de leur ENT, certains étudiants ont néanmoins pu avoir accès aux documents de la plate-forme en utilisant le login d'un camarade, en récupérant les documents imprimés ou encore en travaillant à plusieurs sur un même poste informatique et un même identifiant de connexion.

Par ailleurs, le déploiement d'une telle plateforme permet de s'affranchir d'un besoin de connaissances de sites d'intérêt et/ou de l'utilisation de moteurs de recherche en guidant les étudiants vers des ressources et activités didactiques appropriées.

Le nombre important de ressources mis à disposition est peut-être un frein à une utilisation exhaustive. Toutefois, l'exhaustivité de consultation des différentes ressources n'est pas l'objectif pédagogique de ces cours. Chaque étudiant peut choisir la ou les ressources qui lui conviennent le mieux pour compléter sa formation. Par exemple, les documents complémentaires sont des ressources d'intérêt pour rappeler les bases théoriques ou aller un peu plus loin dans la compréhension de la lecture critique mais peuvent être redondantes avec des livres ou polycopiés éventuellement déjà acquis par les étudiants. Les ressources concernant plus directement le cours peuvent aussi être consultées ponctuellement pour un article moins bien compris ou lors d'une absence au cours en amphithéâtre. Les performances similaires à l'examen final entre utilisateurs réguliers et extensifs de la plate-forme semblent en faveur de ces hypothèses. Néanmoins, notre étude ne permet pas de savoir si la mise à disposition des étudiants d'une plate-forme numérique contenant seulement un nombre limité de documents essentiels n'aurait pas un impact similaire sur les apprentissages.

Certaines ressources innovantes sollicitant plus particulièrement l'interaction des étudiants comme les quiz ou l'accès aux messages sur le forum de discussion n'ont été que rarement utilisées. La nouveauté de ces ressources pour les étudiants ainsi que certaines faiblesses du dispositif pédagogique, notamment un déficit de l'accompagnement des étudiants, pourrait être à l'origine de ce phénomène. La faible consultation du forum peut aussi s'expliquer par l'envoi systématique sur l'adresse courriel de l'étudiant d'une copie des messages postés sur le forum. Toutefois, les étudiants semblent hésiter à poser des questions sur le forum dans lequel tout message déposé est nominatif de l'auteur et conservé durant toute l'année universitaire. Seulement deux questions, dont une concernant l'organisation des enseignements, ont été postées sur le forum alors que les étudiants posent fréquemment des questions à l'issue du cours en amphithéâtre. Ce manque d'interaction diminue l'intérêt des possibilités d'évolutivité du cours en ligne en réponse aux sollicitations des étudiants et aucune ressource didactique supplémentaire n'a été introduite en cours d'année.

Les corrigés types écrits ont été logiquement les ressources auxquelles les étudiants ont eu le plus souvent accès. Ceci peut s'expliquer par le fait que, bien que les cours en amphithéâtre détaillent la réponse à donner aux questions, proposent les motsclés et une réponse type, les méthodes de travail des étudiants leur font préférer l'accès à un document synthétique unique. Cette attitude peut-être considérée comme favorisant des apprentissages superficiels dans le but d'une meilleure réussite aux examens plutôt que des apprentissages en profondeur et transférables. Elle est concordante avec une étude dans une autre université dans laquelle les étudiants déclaraient utiliser le plus souvent une plateforme numérique pour télécharger les notes de cours et consulter des éléments d'information. Toutefois l'observation longitudinale des pratiques a montré une utilisation croissante au cours du temps des activités d'apprentissage qui vont au-delà de la simple réception d'information ${ }^{[13]}$.

Finalement, dans la perspective actuelle d'apprentissage de la médecine tout au long d'une carrière, il est crucial d'acquérir la compétence 
permettant d'appréhender de façon critique l'information scientifique mais il est tout aussi fondamental d'appréhender la nécessité d'acquérir une autonomie de formation. Nos constats, effectués dans le cadre d'un début d'utilisation par les étudiants des ressources numériques asynchrones mises à disposition, soulignent l'étendue du chemin à parcourir pour rendre les médecins autonomes vis-à-vis de leur formation. Ils soulignent qu'une telle autonomie n'est pas une donnée que l'on peut postuler mais une finalité qu'il faut viser chez les étudiants grâce à des interventions pédagogiques appropriées. Ils renforcent la notion établie que la valeur ajoutée d'un dispositif de formation de type hybride est étroitement tributaire de la qualité de la supervision et du support socio-cognitif que les étudiants reçoivent ${ }^{[3]}$. Cette dimension devrait très clairement être optimisée dans le cadre de l'évolution de notre dispositif de formation, à la fois grâce aux interactions étudiantsenseignants lors des séances en présence mais aussi en mettant en place un télé-tutorage.

Les deux indicateurs choisis pour mesurer l'activité des étudiants sur la plate-forme numérique, variété et répétition de l'utilisation, sont proches et fortement corrélés. Globalement un accès varié et/ou répété aux ressources mises à disposition est associé à de meilleures performances lors de l'évaluation sommative des étudiants. Cette tendance n'est pas uniquement expliquée par le niveau général (scores hors LCA en DCEM3) et spécifique (score de LCA en DCEM2) de l'étudiant, puisqu'elle persiste après ajustement sur ces deux variables. Les utilisateurs actifs (réguliers ou extensifs) obtenaient en moyenne entre un demi et un point de plus que les non-utilisateurs ou utilisateurs anecdotiques. Le gain d'un demi-point sur vingt est modéré mais peut être stimulant pour les étudiants et les facultés car pouvant correspondre à plusieurs centaines de places de gagnées lors des ECN. Il est par contre impossible d'apprécier l'impact réel sur les apprentissages, notamment à long-terme, de la lecture critique. Enfin, une explication possible à l'association statistique mise en évidence est que les indicateurs utilisés permettent simplement de repérer les étudiants les plus travailleurs et/ou les plus intéressés par la lecture critique.

Bien qu'il existe une corrélation entre les scores de LCA en DCEM2 et en DCEM3, celle-ci est faible $(r=0,32)$, ce qui illustre que la construction de compétences en LCA doit bien s'envisager de façon graduelle sur la durée et il semble important de stimuler le travail des étudiants afin qu'ils puissent acquérir de bonnes capacités à critiquer les études biomédicales. Cette observation est en accord avec une étude montrant une amélioration des scores au fur et à mesure de la pratique ${ }^{[14]}$. Une des possibilités pour stimuler cette pratique peut être justement le recours à des dispositifs d'enseignement de type hybride, articulant des activités en présence et des activités à distance, dans un environnement médiatisé par les outils pédagogiques numériques.

Cette étude est observationnelle et afin de conclure à l'efficacité du cours mis en ligne sur la plate-forme, on aurait pu conduire un essai randomisé contrôlé comparant des étudiants ayant accès ou non à celui-ci. Si un tel schéma d'étude est parfois utilisé ${ }^{[15]}$, il était pour nous impossible à réaliser car les scores obtenus conditionnent le passage dans l'année d'étude suivante. De plus, les étudiants concernés seront la première promotion à passer l'épreuve de LCA lors des ECN de 2009. Une évaluation pragmatique de la pertinence de l'investissement pédagogique réalisé reste nécessaire. Il sera aussi primordial de suivre la progression de l'utilisation de la plate-forme au fil des promotions.

\section{Conclusion}

$\mathrm{Au}$ total, l'interaction des étudiants avec une nouvelle plate-forme numérique dans le cadre d'un dispositif de formation hybride dédié à la formation à la LCA, même si elle est débutante, est associée à une variation modérée mais positive des scores à l'examen de fin d'année et pourrait avoir un impact positif sur l'apprentissage de la lecture critique d'articles scientifiques. Ces résultats corroborent les résultats déjà obtenus par notre équipe sur un enseignement à distance de l' «evidencebased medicine » utilisant une autre plate-forme 
pédagogique ${ }^{[16]}$. D'autres travaux sont nécessaires pour déterminer plus spécifiquement quelles sont les attentes des étudiants en termes de services numériques pour la pédagogie et quel est l'impact de ces services sur les performances des étudiants et les habitudes de travail et de formation de ces futurs professionnels.

\section{Remerciements}

Nous tenons à remercier Jean-François Pellissier (doyen de la faculté de médecine de Marseille) et André Littolff (centre d'informatique de gestion et réseau, faculté de médecine de Marseille) pour nous avoir facilité l'accès et l'utilisation des résultats d'examens des étudiants, ainsi que Julien Cros (mission technologies de l'information et de la communication pour l'enseignement (TICe), université de la Méditerranée) pour la récupération des traces de connexion.

\section{Contributions}

Julien Mancini a conçu le protocole de recherche, analysé les données, interprété les résultats et rédigé les versions initiale et finale du manuscrit. Roch Giorgi, Jean Gaudart, Jean-Charles Dufour et Marius Fieschi ont conçu le protocole de recherche, interprété les résultats et participé à la révision critique et à l'approbation finale du manuscrit.

Ce travail a initialement fait l'objet du mémoire de Julien Mancini pour le diplôme universitaire de pédagogie médicale 2007/2008 (faculté de médecine de Marseille, université de la Méditerranée).

\section{Références}

1. Cook DA, Levinson AJ, Garside S, Dupras DM, Erwin PJ, Montori VM. Internet-Based Learning in the Health Professions: A Meta-analysis. JAMA 2008;300:1181-96.

2. Cook DA. The failure of e-learning research to inform educational practice, and what we can do about it. Med Teach 2009;31:158-62.
3. Deschryver N. Apprentissage collaboratif en formation hybride. Education-Formation 2009; e-290:79-91. [On-line] Disponible sur : http://ute3.umh.ac. be/revues/include/download. php?idRevue $=6$ \& idRes $=37$

4. Fieschi M, Giorgi R, Gouvernet J, Degoulet P. De la connaissance à la pratique clinique : l'introduction de la médecine basée sur le niveau de preuve. Rev Med Interne 2000;21:105-9.

5. Huguier M. Épreuve de lecture critique d'articles médicaux : une étape importante que les enseignants ne doivent pas manquer. Presse Med 2007;36:565-6.

6. Arrêté du 6 décembre 2007 fixant la pondération de l'épreuve de lecture critique d'articles lors des épreuves classantes nationales du troisième cycle des études médicales, NOR : ESRS0772655A. Journal Officiel de la République Française du 20 décembre 2007, texte 50:20518. [On-line] Disponible sur : http://www. legifrance.gouv. fr/jopdf/common/jo_pdf. jsp?num $0=0 \&$ date $J 0=20071220$ \&numTexte $=50$ \& pageDebut $=20518 \&$ pageF in $=20518$

7. Conseil scientifiques des concours d'internat -CNCI. Épreuve de lecture critique d'article médical. 2007. [On-line] Disponible sur : http://www.cnci.univparis5.fr/medecine/LectureCritiqueArticle. pdf

8. ANAES/Service Recommandations Professionnelles. Guide d'analyse de la littérature et gradation des recommandations, Paris : ANAES 2000.[On-line] Disponible sur : http://www.has-sante.fr/portail/jcms/ c_434715/guide-danalyse-de-la-litteratureet-gradation-des-recommandations

9. Cucherat M. Guide de lecture critique d'un essai thérapeutique. Médecine Thérapeutique 2006;12:354-8.

10. Doll H, Carney S. Les approches statistiques de l'incertitude : tout ce que vous avez toujours voulu savoir sur les valeurs de $\mathrm{p}$ et les intervalles de confiance. EBM journal 2006;46:9-11.

11. Straus SE, McAlister FA. Guide à l'usage du clinicien sur les études pronostiques publiées dans la littérature. EBM journal 2000;21:4-6.

12. Ricard JD, Lejoyeux M, El-Ghoneimi A, Matheron S, Maillard D, Crickx B, et al. Utilisation des nouvelles technologies de l'information et de la communication par les étudiants en médecine. Enquête de pratique et mise en situation. Pédagogie Médicale 2005;6:112-122.

13. Docq L, Lebrun M, Smlidts D. A la recherche des effets d'une plateforme d'enseignement/apprentissage en ligne 
sur les pratiques pédagogiques d'une université : premières approches. Revue internationale des technologies en pédagogie universitaire 2008;5:45-57.

14. François P, Boyer L, Nuiry L, Labarere J. Évaluation des propriétés docimologiques d'une épreuve de lecture critique d'articles médicaux scientifiques. Presse Med 2007;36:575-81.

15. Bodjrenou AJ, Kouawo AC, Moumouni H, Darboux R. Évaluation de l'impact de la mise en ligne d'un programme d'histologie-embryologie sur les performances aux examens d'étudiants en première année d'études médicales au Niger. Pédagogie Médicale 2008;9:181-4.
16. Fieschi M, Soula G, Giorgi R, Gouvernet J, Fieschi D, Botti $\mathrm{G}$, et al. Experimenting new paradigms for medical education and the emergence of a distance learning degree with the Internet: Teaching Evidence Based Medicine. Med Inform Internet Med 2002;27:1-11.

Correspondance et offprints : Dr Julien Mancini, Laboratoire $\mathrm{d}^{\prime}$ Enseignement et de Recherche sur le Traitement de l'Information Médicale (LERTIM, EA 3283), Faculté de médecine de Marseille, 27 Bd Jean Moulin, 13385 Marseille Cedex 05, France Mailto : julien.mancini@univmed.fr 